\title{
¿COLOMBIA DEBE APOSTARLE AL SOFTWARE LIBRE, AL DE FUENTE ABIERTA O AL PROPIETARIO?
}

\author{
Luis Alberto Girón Murillo \\ Universidad Autónoma de Bucaramanga \\ Director Ejecutivo Corporación Educativa Ser y \\ Hacer Medellín \\ montaneros@gmail.com
}

\author{
Jorge Andrick Parra Valencia \\ Universidad Autónoma de Bucaramanga \\ Presidente Comunidad Colombiana de \\ Dinámica de Sistemas \\ andrick.parra@gmail.com
}

(Tipo de Artículo: Investigación. Recibido el 20/01/2014. Aprobado el 10/05/2014)

\begin{abstract}
RESUMEN
Es complejo para gestores y administradores conocer las implicaciones que la adopción de modelos de desarrollo de software tendría para una región. Este artículo ofrece una evaluación de las implicaciones dinámicas que para el desarrollo de software de una región tendría el apostar de forma excluyente al software libre, al software de fuente abierta y al software propietario. Se desarrolló un modelo de simulación en Vensim de la dinámica de sistemas en donde se evaluaron 3 escenarios: uno en donde se adopta de forma excluyente el software libre, uno en donde se adopta el software propietario y uno en donde se adopta el software de fuente abierta. Se ofrecen descripciones y explicaciones de los resultados que sugieren las potencialidades de cada uno de los modelos de desarrollo evaluados.
\end{abstract}

\section{Palabras clave}

Pertinencia del software, dinámica de sistemas, software libre, software propietario, software de fuente abierta, modelos de simulación.

\section{Must Colombia Opt for Free, Open or Proprietary Software?}

\begin{abstract}
This paper is intended for evaluating, by means of simulation models, the dynamic implications that would have for the development of Medellin software industry opting exclusively for free software, opting for open source software and opting for proprietary software in order to improve the planning and design of policies for this sector. This article also presents the design of a simulation model in Vensim about the dynamic of systems which evaluates and compares through simulations the increase in implications such as: software developer training, initiation and development of software applications, production of developed applications and the influence of these applications in the productivity of individuals and companies, and the emergence and stabilization of productive companies in the region, which will provide elements of judgment when opting for any of these types of software
\end{abstract}

\section{Keywords}

Pertinence of the software, System Dynamics, Free Software, Proprietary Software, Simulation models.

\section{¿LA COLOMBIE DOIT-ELLE SE DÉCIDER POUR LOGICIELS LIBRES, LOGICIELS DE SOURCE OUVERTE OU POUR LOGICIELS PROPRIÉTAIRES?}

\begin{abstract}
Résumé. Cet article a comme objectif d'évaluer, en utilisant de modèles de simulation, les implications dynamiques qui résultent pour l'industrie des logiciels de la ville de Medellin, Colombie, le choix d'une manière exclusive des logiciels libres, des logiciels ouvertes et des logiciels propriétaires pour l'amélioration du planning et conceptions de politiques pour le secteur. On présente la conception d'un modèle de simulation sur le logiciel Vensim de la dynamique des systèmes où sont évalués et comparés l'accroissement dans implications comme: formation de développeurs de logiciels, initiation et développement d'applications pour logiciels, production des applications développés et linfluence de ces applications sur la productivité des personnes et des entreprises, et sur la naissance et la stabilisation des entreprises productives de la région, qui vont apporter des éléments de connaissance pour se décider pour une des types de logiciels déjà mentionnés.
\end{abstract}

Mots-clés. Pertinence des logiciels, Dynamique de systèmes, Logiciels libres, Logiciels propriétaires, Modèles de simulation. 


\section{INTRODUCCIÓN}

Con el pasar del tiempo se ha evidenciado un vertiginoso avance de las ciencias y las Tecnologías de la Información y la Comunicación (TIC), la cual ha permitido los múltiples escenarios y formas de abordar los distintos procesos en el manejo de los datos (información). En Colombia, al igual que en la mayoría de los países del mundo, se ha venido utilizando distintas clases de software (propietario, libre y de código abierto), que se emplea en el manejo de la información que generan sus distintos procesos. Colombia presenta una industria de software con serios problemas de articulación, es decir desarticulada, con muchísimo camino por recorrer aunque se viene trabajando fuertemente por tratar de agremiar las empresas de software y su articulación con el estado, aun no existen cifras confiables de importaciones ni exportaciones de software porque el país no ha avanzado en la clasificación de intangibles para la contabilidad del comercio exterior. No es posible obtener acceso a cifras reales del comercio exterior ni mucho menos a tendencias históricas del software en Colombia y tampoco hay información sobre la distribución del mercado, es decir una cifra real para decir que porcentaje de cada uno de los modelos de software es el que se está usando en Colombia [1].

Según una encuesta realizada a las empresas colombianas, el $75 \%$ de las empresas encuestadas dijeron que si estaban utilizando software libre [2]. Lo que confirma que se está usando, pero no es publicado lo que hacen, es decir, no se comparte información. Son pequeñas islas donde cada quien tira para su lado.

Colombia en materia de gobierno ha venido fijando sus ojos con timidez en el software Libre, que según la Tendencias relevantes en informática es: servicios Web, negocios electrónicos, computación en malla, computación autonómica, aplicaciones móviles, software libre y de código abierto, seguridad informática. Lo que deja ver que se pretende invertir en esta materia [3].

Pero al revisar "Plan Vive Digital", que establece diversas iniciativas para masificar el uso de Internet en Colombia, se observa que el programa Compartel pretende implementar una serie de nuevos proyectos que buscan la masificación del uso de Internet con el fin de contribuir a generar empleo, reducir la pobreza y hacer a Colombia más competitiva, con programas como: Proyecto Nacional de Fibra Óptica, Proyecto Puntos Vive Digital, Proyecto Hogares Digitales, Proyecto de Acceso a las TIC en Zonas Rurales y/o Apartadas [4], los cuales le apuestan a que sean implementados a través de operadores privados, y sin hacer referencias a tecnologías libres en el caso del uso de software libre ni código abierto. Por lo anterior se puede decir que Colombia no es un país que saca ventaja en materia de aprovechamiento respecto al uso del software Libre y código abierto, lo cual se refleja al ocupar el puesto 45 de 75 en el índice de código abierto [5].

Colombia todavía cuenta con problemas en las transferencias tecnológicas especialmente en el ámbito del software libre y código abierto, que se explica con la ausencia de decisiones gubernamentales en un modelo que necesita de ellas para poder impactar el mercado masivo [6].

En cuanto a los modelos de producción de software, se puede decir que existen variedades que se diferencian en las formas de concebir el software, ya que el resultado de aplicarlo, siempre conduce a lo mismo, que es producir software de buena calidad; está el modelo de producción de software de código abierto, que tiene como una de las razones por las cuales este modelo descentralizado funciona tan bien es que el software es modular. Es decir, se puede construir por partes, con pequeños grupos dedicados a distintas pequeñas partes del mismo [2].

Aplicar modelos de producción de software en Colombia es algo que no está a la luz, ya que las producciones son casi ocultas y quienes las están haciendo las mantienen en este estado, "solo para el que paga", es otra de las desventajas de Colombia en la producción de software, ya que no cuenta con un departamento de publicidad [7], poco se comparte lo que se hace, sin aplicar el concepto de libere rápido y a menudo y delegue lo que pueda, llevado al nivel de la promiscuidad al ser abierto, que es lo primordial en los modelos de producción del software libre y de código abierto [8].

El termino software libre se refiere a las 4 libertades que deben tener los usuarios para ejecutar, copiar, distribuir, estudiar, cambiar y mejorar dicho software, es decir [9]. Libertades que se encuentran en el documento que garantiza que un software es y seguirá siendo libre, como es la Licencia Publica General, conocida como la GPL [10]. El concepto de software libre como tal no aparece hasta principios de la década de 1980, que es donde se comienza hablar del software Libre, término que no era usado porque no existía [11].

El Software propietario, que es uno de los términos utilizados para denominar a cualquier programa o software informático que no es libre, o que su código fuente no está disponible o el acceso a este se encuentra restringido [12]. La expresión software propietario proviene del término en inglés "proprietary software", que reserva los derechos sobre el uso, modificación o redistribución del software [13]. El software propietario es aquel que a su creador, es decir, a su desarrollador, el sistema de derechos de autor le reconoce y le garantiza un monopolio de explotación de sus obras [14]. 
La industria del software. Es la industria que involucra la investigación, desarrollo, distribución y comercialización de software, sin tener en cuenta el tipo al cual pertenece, ya sea libre, abierto o privativo. En Colombia las empresas de software están distribuidas. Empresas de Instalación y Comercialización, empresas de Consultoría de TI, empresas dedicadas al desarrollo de software a la medida, empresas de implementación de software y empresas dedicadas a prestar servicios de soporte [15].

Dinámica de Sistemas es la designación de un método que se utiliza para construir modelos de sistemas sociales susceptibles de que sean simulados a través de un computador. Esta técnica que se inicia en los años cincuenta y su desarrollo se da en la década de los sesenta, gracias a los trabajos de J. W. Forrester del Instituto Tecnológico de Massachussets [16]. Los Modelos de Dinámica de Sistemas representan la estructura de realimentación del sistema, y la simulación del modelo muestra el efecto de la intervención política en la estructura del sistema [17].

En este artículo se evalúa mediante un modelo de simulación con tres escenarios de software las implicaciones dinámicas que tendría para el desarrollo de la industria de software de Medellín el apostarle de forma excluyente al software libre, al software de fuente abierta y al software propietario para el mejoramiento de la planeación y diseño de políticas para el sector, en el cual se diseña y se describe un modelo de simulación de la dinámica de sistemas con tres escenarios de software en los cuales se comparan y se describen a través de simulaciones las implicaciones o variables tales como: "Desarrolladores de software, Aplicaciones en desarrollo, Aplicaciones desarrolladas y Empresas productivas de la región", mostrando en cuál de los tres escenarios de software del modelo se maximiza mejor de estas variables.

El modelo se describe a través de 3 bloques, así: Un primer bloque conformado por la variable de nivel Desarrolladores de software. Un segundo bloque conformado por las variables de nivel Aplicaciones en desarrollo y Aplicaciones de software desarrolladas. Finalmente se tiene el tercer bloque, la cual está formado por la variable de nivel Empresas productivas de la región.

El artículo se organiza de la siguiente manera: en la sección 2 se presenta el modelo y su descripción, en la sección 3, se describen los resultados obtenidos en las simulaciones del modelo, en la sección 4 aparecen las discusiones con otro trabajos, en la sección 5 aparecen los aportes novedosos, en la sección 6 aparecen las conclusiones, en la sección 7 aparen los trabajos futuros y en la sección 8 las referencias.

\section{EL MODELO}

Para el desarrollo de este trabajo se ha utilizado una metodología dividida en dos procesos: la revisión bibliográfica y el modelado del prototipo, objeto de esta investigación junto con su posterior análisis.

Para la revisión y análisis de la bibliografía, se ha llevado a cabo una búsqueda de artículos y documentos sobre los diferentes paradigmas implicados en la investigación y que se han especificado en los objetivos de este trabajo. De una parte se han estudiado referencias de trabajos relativos a la gestión e inversión en materia de software libre, propietario, fuente abierta y la industria del software en Colombia

Se ha realizado una concienzuda revisión bibliográfica de publicaciones científicas y tecnológicas referidas a los avances de los sistemas de información, haciendo especial énfasis en el estudio de los modelos de software. Finalmente se han examinado las principales publicaciones referidas al área de la dinámica de sistemas.

En cuanto al proceso de modelado y análisis se ha seguido la metodología propia de la Dinámica de Sistemas, para lo cual tenemos los siguientes pasos:

Identificación del problema y análisis del comportamiento. Se determinan las variables clave, se definen los límites del modelo y se describen los modos de referencia que son los comportamientos que caracterizan al sistema.

Modelado causal del sistema. Se elaboran las hipótesis dinámicas, o causal, las cuales incluyen las influencias detectadas entre los elementos del sistema, y así obtener el Diagrama de flujo o, de Forrester.

Modelado de Forrester. Se realiza la transformación o paso al Diagrama de flujos y niveles, el cual es simulado en un computador, obteniendo así, las ecuaciones matemáticas que definen el comportamiento del sistema y especificando las diferentes variables, las magnitudes 0 unidades, el alcance, la frecuencia de simulación y el horizonte temporal

Validación del modelo. Se verifica la sintaxis y coherencias dimensionales de las ecuaciones que constituyen el diagrama de flujo y niveles, se valida que el modelo "diagrama de Forrester" responda al comportamiento especificado y se analiza la sensibilidad del modelo en las distintas simulaciones realizadas.

La Herramienta de Simulación Utilizada. Para la construcción del diagrama de Forrester y posteriores simulaciones, se ha utilizado uno de los muchos 
entornos de simulación existentes de la Dinámica de Sistemas, como es Vensim $\AA$ que es un software integrado que proporciona un entorno gráfico que permite conceptualizar, documentar, simular, analizar y optimizar modelos de Dinámica de Sistemas. Se utilizó Vensim®PLE en la versión para estudiantes que se descarga de (http://vensim.com/) concretamente la configuración utilizada es Vensim $\AA$ PLE Plus for Windows Versión 5.11A.

\subsection{Resultados alcanzados}

La realización de esta investigación, permite obtener resultados en dos aspectos muy importantes, tales como:

Una revisión de la literatura sobre el impacto en el desarrollo de la industria de software de los modelos de desarrollo enfocados al software libre, al software de fuente abierta y al software propietario.

Un modelo de simulación con tres escenarios de software que permiten evaluar mediante simulación las implicaciones dinámicas que para la industria del software en Colombia tendría la adopción excluyente de modelos de desarrollo enfocados al software libre, al software de fuente abierta y al software propietario, que contribuyan al mejoramiento de la planeación y diseño de políticas para el sector.

\subsection{Aportaciones}

Esta investigación aporta a la ciencia una revisión y análisis de la literatura existente sobre el impacto en el desarrollo de la industria de software de los modelos de desarrollo enfocados al software libre, al software propietario y de la dinámica de sistemas.

También aporta un modelo de simulación con tres escenarios de software (Libre, propietario y fuente abierta), realizado en Vensim de la dinámica de sistemas, en el cual se evalúan y se comparan unas implicaciones o variables (Desarrolladores de software, Aplicaciones en desarrollo, Aplicaciones desarrolladas y Empresas productivas de la región) en los tres escenarios mediante simulaciones y la descripción de los resultados de estas simulaciones.

\subsection{Bloques básicos del modelo}

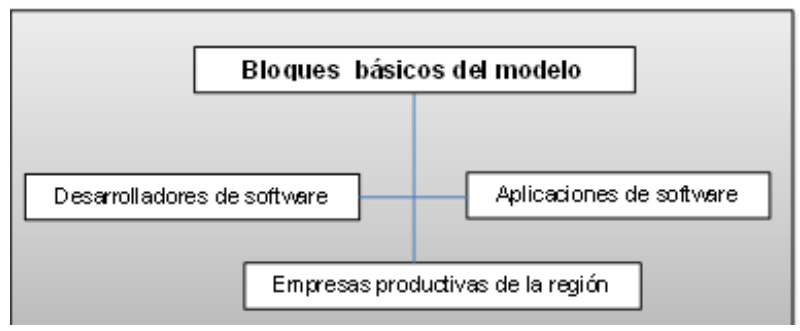

Fig. 1. Bloques básicos del modelo. Fuente: Elaboración propia.

\subsection{Descripción del modelo}

El siguiente modelo es la combinación de una serie de ecuaciones, variables y otros elementos que se han integrado para su elaboración, el cual se describe a continuación a través de tres bloques; y que se observa en la figura 2.

\subsubsection{Bloque uno. Desarrolladores de software}

El primer bloque identificado en el modelo propuesto se refiere a la cantidad tomada como muestra de los desarrolladores de software existentes en la región, los cuales se forman durante un tiempo determinado. Algunas de las variables que intervienen para su cálculo en el modelo son: formación desarrolladores, Retiro de desarrolladores y Desarrolladores de software.

El cálculo de los desarrolladores en formación se obtiene mediante la ecuación número 1. La unidad de medida está dada en persona/mes.

$$
\begin{aligned}
& \text { formación desarrolladores } \\
& =\text { desarrolladores de software } \\
& \text { * multiplicador prodrel vocaciones } \\
& \quad \text { (productividad empresarial relativa) }
\end{aligned}
$$

Para el cálculo de los desarrolladores de software se obtiene restando de la variable formación desarrolladores que se refiere a las personas que por vocación inician la formación como desarrolladores de software o porque simplemente la profesión de desarrollador se muestra en el mercado laboral como una opción atractiva y rentable a la hora de elegir una profesión.

Y se le resta la variable Retiro de desarrolladores, que se refiere a la cantidad de estos desarrolladores que por algún motivo abandonan la profesión, ya sea por retiro forzoso o por desinterés en el tema.

Y así resulta la cantidad de personas formadas como desarrolladores de software de la región por mes en el modelo.

Para calcular los Desarrolladores de software, se utiliza la ecuación número 2. La unidad de medida está dada en personas:

Desarrolladores de software $=$
formación desarrolladores -
retiros desarrolladores

El bloque uno se refleja en la Fig. 3. 


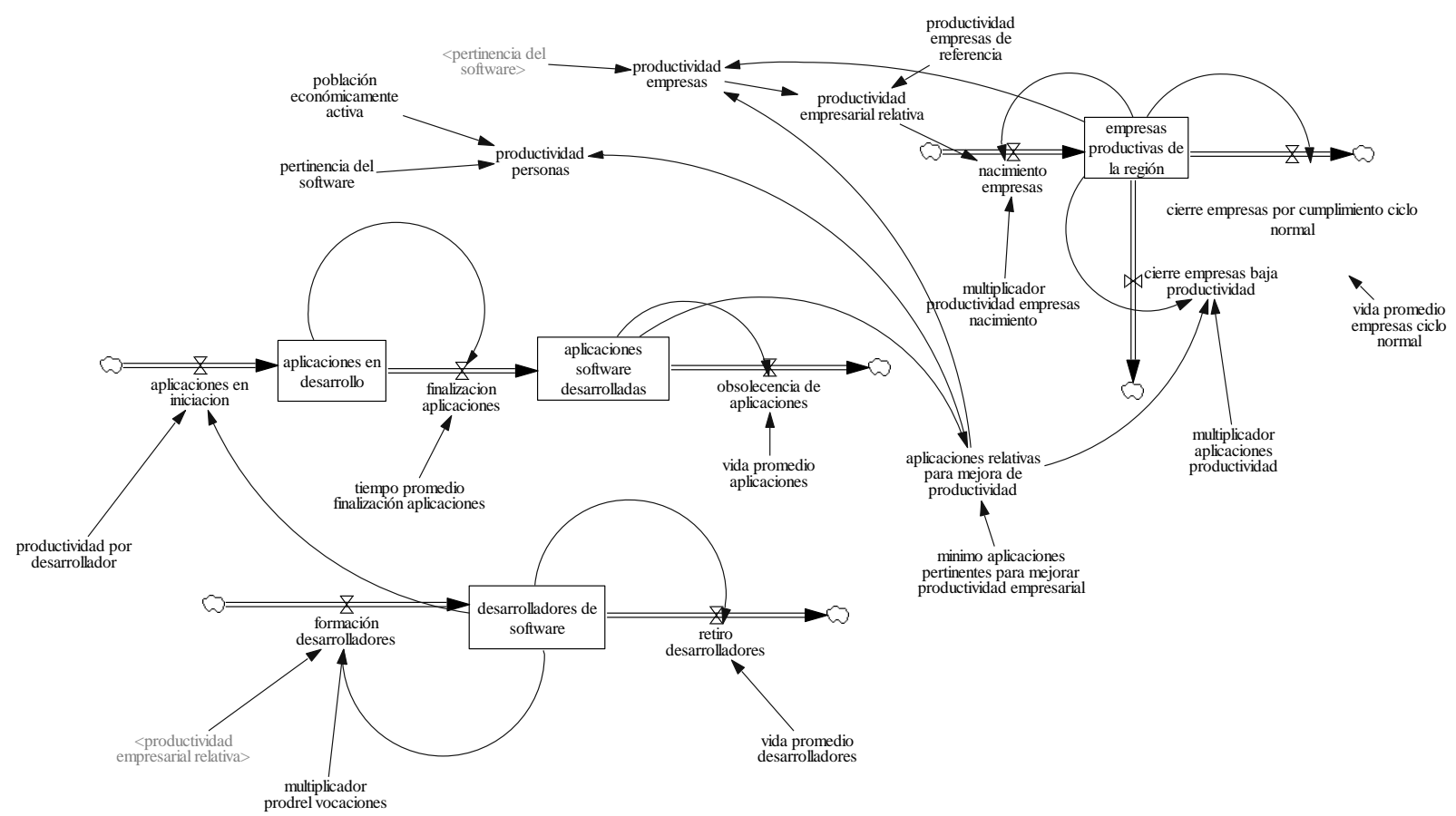

Fig. 2. El Modelo de escenarios de software completo

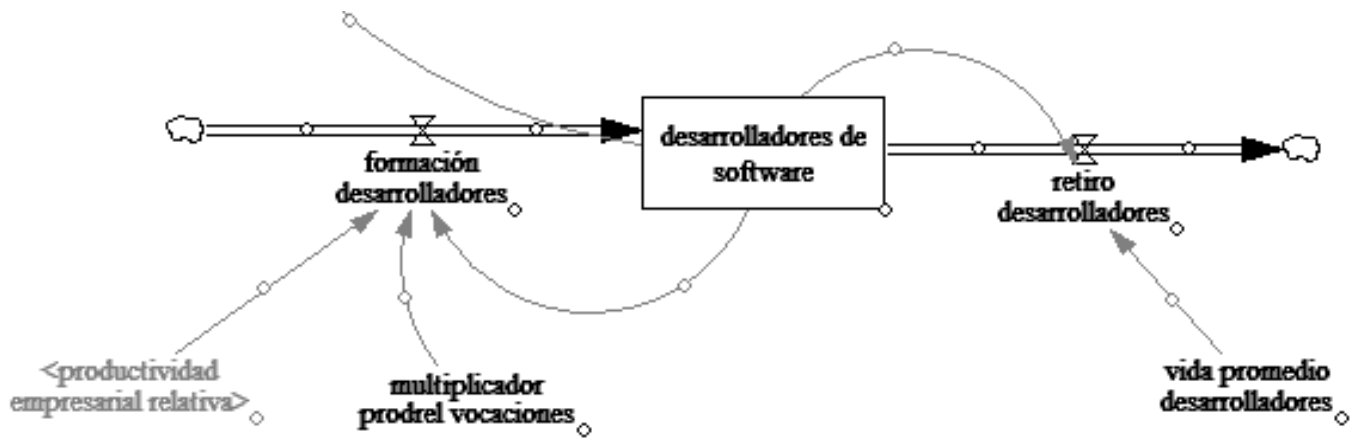

Fig. 3. Bloque uno. Desarrolladores de software

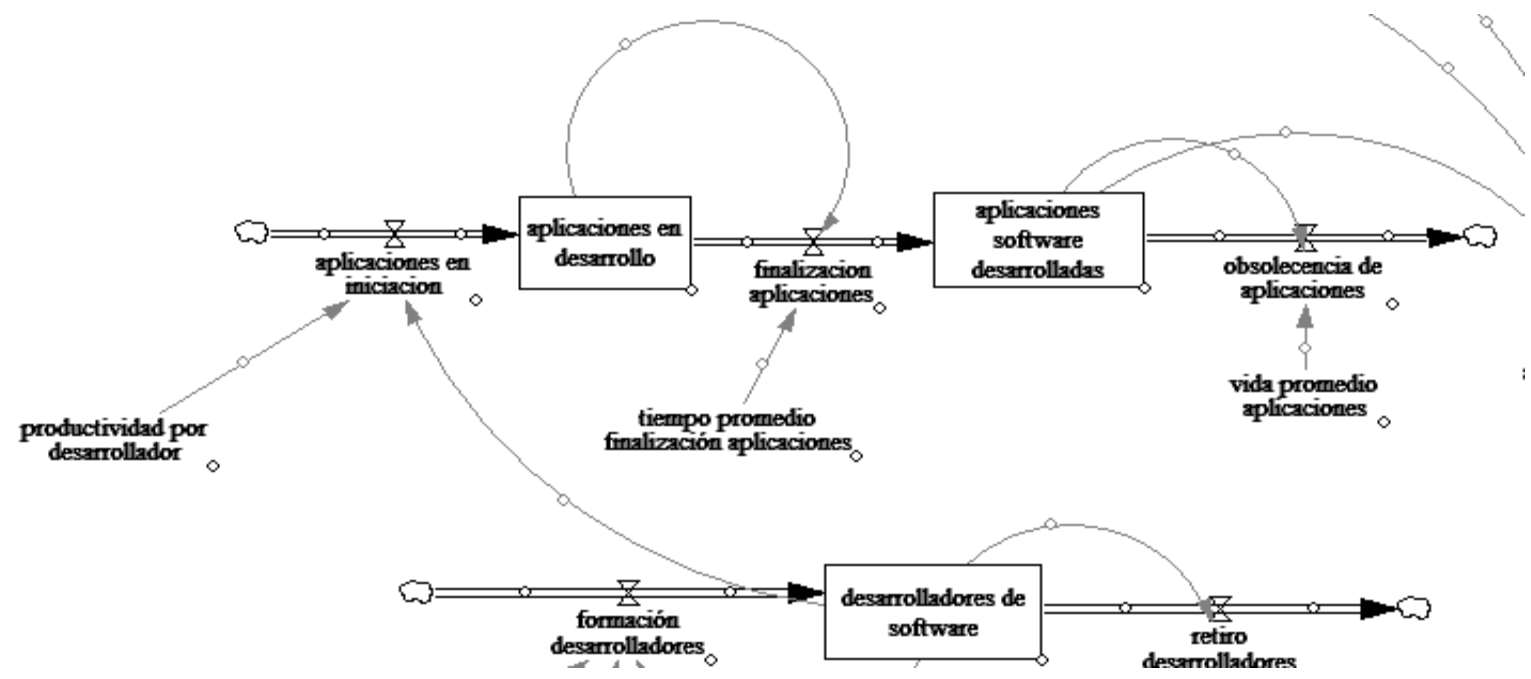

Fig.4. Bloque dos. Aplicaciones de software 


\subsubsection{Bloque dos. Aplicaciones de software.}

El segundo bloque del modelo está formado por las variables de nivel aplicaciones en desarrollo y aplicaciones de software desarrolladas, por las variables de transportes Aplicaciones en iniciación, Finalización de aplicaciones, Obsolescencia de aplicaciones, y por las variables auxiliares Productividad por desarrollador, Tiempo promedio finalización de aplicaciones y vida promedio de aplicaciones. Este bloque que se inicia con el cálculo de las aplicaciones en iniciación, que resulta de multiplicar Productividad por desarrollador por el número de Desarrolladores de software. Luego estas aplicaciones pasan a la etapa de aplicaciones en desarrollo, que resulta de restar las aplicaciones en iniciación menos las aplicaciones finalizadas y así resulta la cantidad de aplicaciones que en el momento se están desarrollando. La ecuación para el cálculo de las aplicaciones en desarrollo es la número 3 y la unidad de medida está dada en aplicaciones.

\section{Aplicaciones en desarrollo $=$ aplicaciones en iniciación - finalización aplicaciones}

Luego esta las aplicaciones de software desarrolladas, que son las aplicaciones que están listas y disponibles para ser utilizadas por los usuarios, las cuales resultan del cálculo de las aplicaciones que salen de la etapa de finalización menos las aplicaciones que han cumplido el ciclo útil, es decir que han pasado a la etapa de obsolescencia. Dando como resultado un listado de aplicaciones útiles y disponibles en el mercado que contribuyen al rendimiento y mejoramiento de la productividad de las personas y las empresas como tal. Para el cálculo de las aplicaciones de software desarrolladas, se utiliza la ecuación número $4 \mathrm{Y}$ la unidad de medida esta expresada en aplicaciones.

\section{Aplicaciones software desarrolladas $=$ finalización aplicaciones - obsolescencia de aplicacione}

El bloque dos se refleja en la Fig. 4.

\subsubsection{Bloque tres. Empresas productivas de la región}

El tercer bloque del modelo, consta de la variable de nivel Empresas productivas de la región y la influencia de las aplicaciones desarrolladas en la productividad. Donde se observa que las empresas productivas de la región son afectadas doblemente por las aplicaciones desarrolladas, dependiendo de su productividad y pertinencia.

Luego se tiene que las aplicaciones de software desarrolladas, una vez están listas y siendo utilizadas, se entra a medir su grado de pertinencia, para lo cual se le da un valor en una escala de cero a uno, siendo cero la menor pertinencia y uno la máxima. Esta pertinencia de las aplicaciones ayuda a mejorar la productividad tanto de las personas como de las empresas. Para medir la productividad de las personas, se toma la población económicamente activa y se multiplica por la pertinencia de las aplicaciones, es decir por el software que comúnmente usa en sus actividades cotidianas multiplicado por las aplicaciones relativas para mejora de productividad, expresado en la unidad de medida persona. La ecuación es:

\section{aplicaciones relativas para mejora de productividad pertinencia del software* población económicamente activa

Para medir la productividad de las empresas, se toma las empresas productivas de la región y se multiplica por la pertinencia del software que es usado en la empresa multiplicado por las aplicaciones relativas para mejora de productividad, generando así el cálculo de la productividad de las empresas, expresado en la unidad de medida empresas y calculado mediante la ecuación:

\section{aplicaciones relativas para mejora de productividad * empresas productivas de la región * pertinencia del software

Luego tenemos en el modelo la variable de flujo de material nacimiento de empresas, la cual está calculada por la productividad empresarial relativa (que es una viable que resulta de multiplicar la productividad de las empresas de referencias, que en este caso se toma una cantidad constante multiplicado por la productividad de las misma empresas), multiplicado por las empresas productivas de la región por el multiplicador productividad empresas nacimiento, generando el cálculo de las nuevas empresas que pueden nacer durante un mes, expresado en la unidad de medida empresa/mes, y la ecuación es:

$\left(\begin{array}{c}\text { empresas productivas de la región } * \\ \text { multiplicador productividad empresas nacimiento } \\ \text { (productividad empresarial relativa) }\end{array}\right)$ (7)

Luego está la variable de nivel empresas productivas de la región, que representa las empresas activas que efectivamente están atendiendo y produciendo en la región. Este dato de las empresas activas en la región se obtiene del cálculo de las empresas que mensualmente nacen en la región impulsadas por la pertinencia de las aplicaciones de software, la cual se convierte en un factor determinante en la productividad de estas empresas y de las personas. Luego a estas empresas que nacen se les resta o descuentan las empresas que cierran o terminan sus operaciones, ya sea porque cumplieron su ciclo normal de actividad o porque su baja productividad no les permite seguir operando en el mercado, en donde también tiene influencia las aplicaciones de software, ya que si las aplicaciones de software utilizadas en la región tienen un alto grado de pertinencia, esta pertinencia va a incidir positivamente en la productividad de las empresas lo que le va ayudar a 
aumentar sus operaciones y permanecer en el mercado, pero si su pertinencia es baja, su incidencia en la productividad en las empresas es baja o negativa por ende sus operaciones son mínimas de forma que difícilmente permanecerán en el mercado, es decir, el cierre por baja productividad es inminente. La ecuación del cálculo de las empresas productivas de la región en el modelo es:

\section{nacimiento empresas - cierre empresas baja productividad - cierre empresas por cumplimiento ciclo normal (8)}

La cual está expresada en la unidad de medidas empresas y se refleja en la Fig. 4.

\section{RESULTADOS OBTENIDOS}

Dado que este proceso iterativo es muy extenso, solo se mostrará el desarrollo de las siguientes actividades para el mejor resultado obtenido:

\subsection{Análisis de los resultados del modelo}

En el modelo se presentan los tres escenarios, el del software Libre, el del software Propietario y el del software de fuente abierta, realizado mediante el sistema de simulación Vensim Software, versión 5.11A; en los cuales se mide el grado de pertinencia, para demostrar cuál de los tres maximiza más las variables del modelo donde se le compare, en un periodo de tiempo de cero a cien $(0$ - 100) meses, y así tener elementos de juicio para decidirse por alguno de los tres.

\subsection{Simulación}

En la simulación, se le ha asignado en la escala de valoración de la pertinencia al escenario del software libre una pertinencia de 0,9 grados, al escenario de software propietario una pertinencia de 0,4 grados y al escenario de software de fuente abierta una pertinencia de 0,3 ; donde ya se indicó que la escala de valores de pertinencia es de cero a uno $(0-1)$, siendo cero la mínima pertinencia y uno la máxima.

\subsubsection{Formación Desarrolladores}

En la figura 6, formación de desarrolladores, se observa que las primeras personas que inician a formarse como desarrolladores en la simulación, sólo aparecen a partir del mes 28 en el escenario de software Libre, mientras que en el escenario de software propietario solo se observa el inicio a partir del mes 52 y en el escenario del software de fuente abierta solo aparecen a partir del mes 66. Luego se ve que en los meses siguientes hay una separación amplia en la cantidad de personas en formación, a tal punto que en el mes setenta (70) mientras que las personas en formación como desarrolladores en software libre ya va en aproximadamente veinte (20) por mes, los de software propietario lo hacen un poco más lento, es decir, solo va en aproximadamente seis (6) y los de software de fuente abierta solo aparecen en una aproximación de dos (2) desarrolladores por mes. $Y$ al llegar al final del tiempo previsto para la simulación que es 100 meses, se observa que el aumento en formación como desarrolladores en software libre sigue siendo mayor, es decir ha alcanzado 80 desarrolladores por mes, mientras que los de software propietario solo alcanzó aproximadamente 21 desarrollador, y los desarrolladores de software de fuente abierta solo llegaron aproximadamente a 9 por mes.

En resumen, lo anterior indica que la base de desarrolladores de software de la región, estará conformada por un número mayor de desarrolladores en software Libre, lo cual se refleja en la figura 6 , en donde se presentan los tres escenarios y su producción en formación de desarrolladores, concluyendo que el software libre por tener la mayor pertinencia atrae el mayor número de personas que empiezan a formarse como desarrolladores potencializando así la variable Formación Desarrolladores la cual indica que como respuesta parcial a la pregunta de investigación Colombia debe apostarle al software Libre en materia de formación.

\subsubsection{Desarrolladores de software}

En los inicios de la simulación del modelo con la pertinencia del software libre en 0,9 , el software propietario en 0,4 y el software de fuente abierta en 0,3, se observa en la figura 7, Desarrolladores de software, que las líneas de ambos escenarios es muy pareja, a tal punto que nada más se ve una línea porque no hay diferencias en ambas, solo hasta aproximadamente el mes 37, empiezan a separarse, es decir, hasta el mes 37 la formación de desarrolladores es pareja en ambos escenarios, aproximadamente 125 desarrolladores de software se han formado en cada uno de los escenarios. Luego a partir de este momento (mes 37), la línea del escenario de software libre se separa y toma una gran ventaja a sus competidores con respecto al incremento en desarrolladores formados, como se observa en la figura 7. Esto ratifica y es coherente con lo sucedido en la etapa de formación de desarrolladores de software de la región, que según la Fig. 6, formación de desarrolladores, que la mayoría lo están haciendo en software Libre, como se observa al final de la simulación en la figura. 7, en el mes 100, que la diferencia entre ambos es muy amplia, mientras que la línea de software Libre alcanza aproximadamente las 1600 personas formadas como desarrolladores, la de software propietario solo alcanza aproximadamente 400 desarrolladores y en el escenario de software de fuente abierta, solo se alcanzaron a formar 200 personas aproximadamente como desarrolladores de software.

En resumen, al observar lo sucedido en la figura 6, en donde se muestra que el mayor número de personas en formación corresponde al escenario de software libre, lo cual es coherente con los resultados obtenidos de la variable Desarrolladores de Software, como se ve en la figura 7, que la mayoría son personas formadas como desarrolladores en software Libre. 


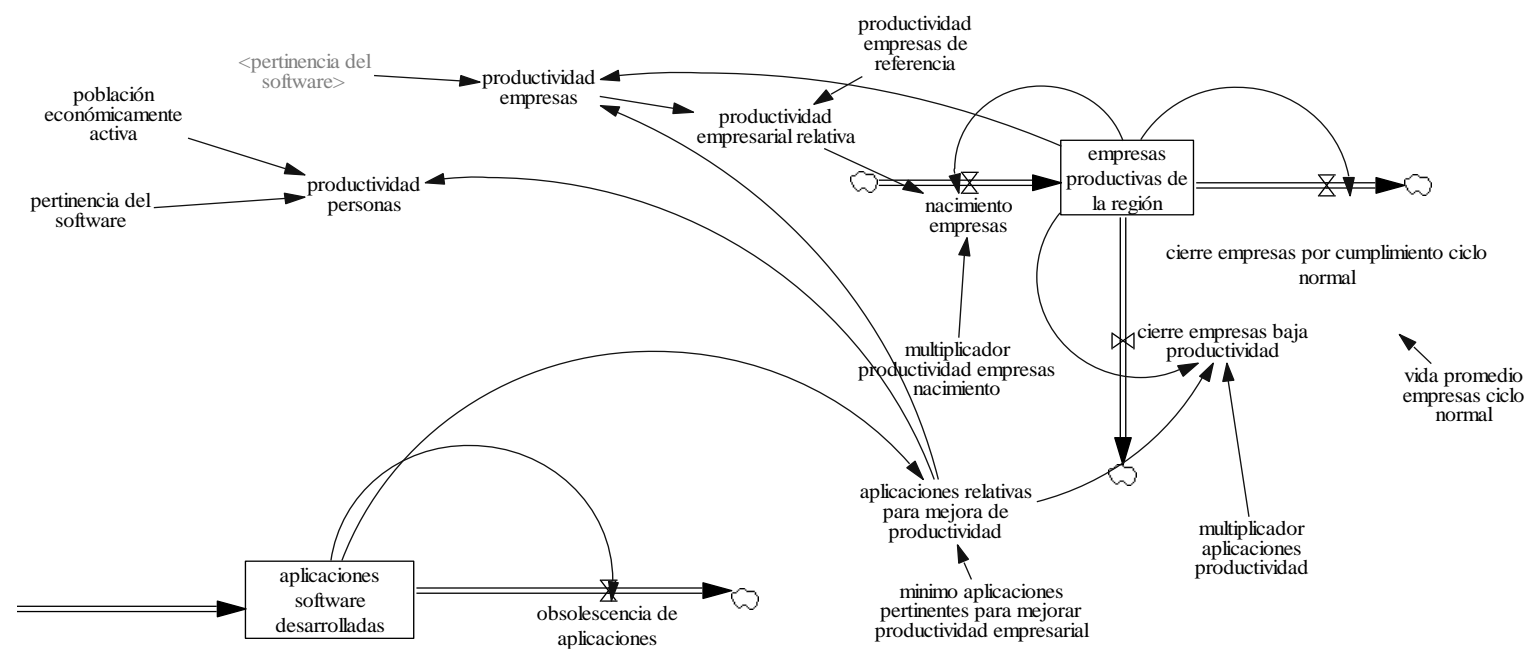

Fig. 5. Bloque tres empresas productivas de la región

formación desarrolladores

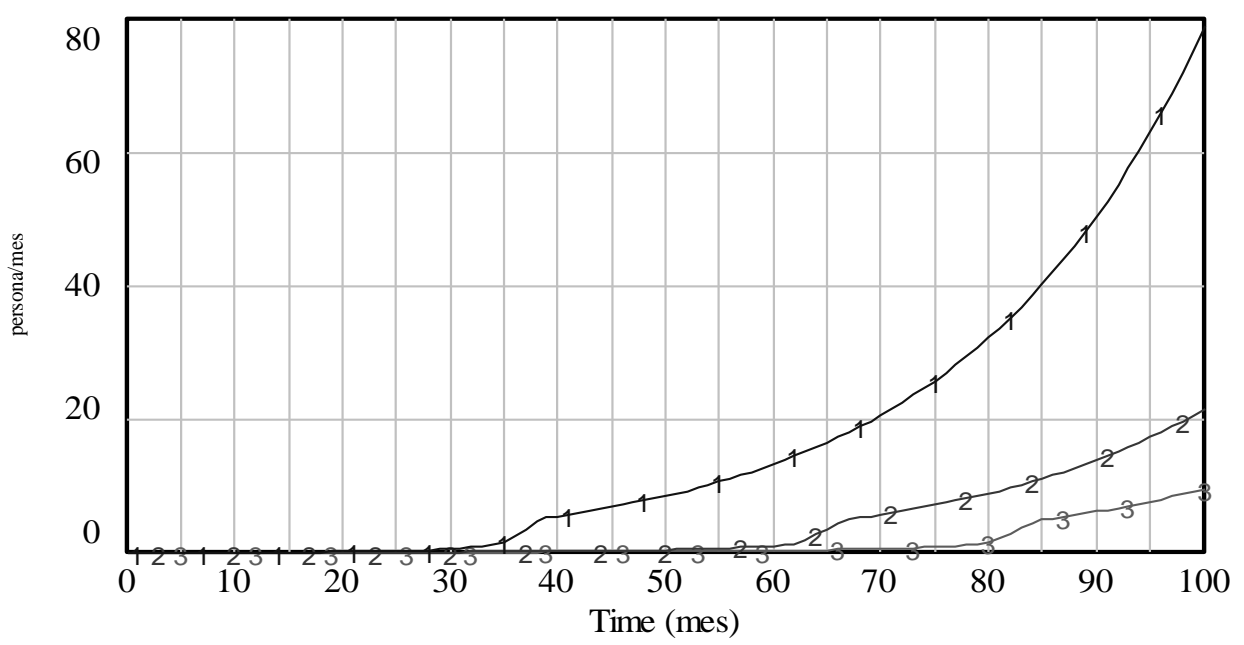

formación desarrolladores : escenario software libre 9
formación desarrolladores : escenario software propietario4
formación desarrolladores : escenario software fuente abierta Fig. 6. Formación de desarrolladores

Desarrolladores de software

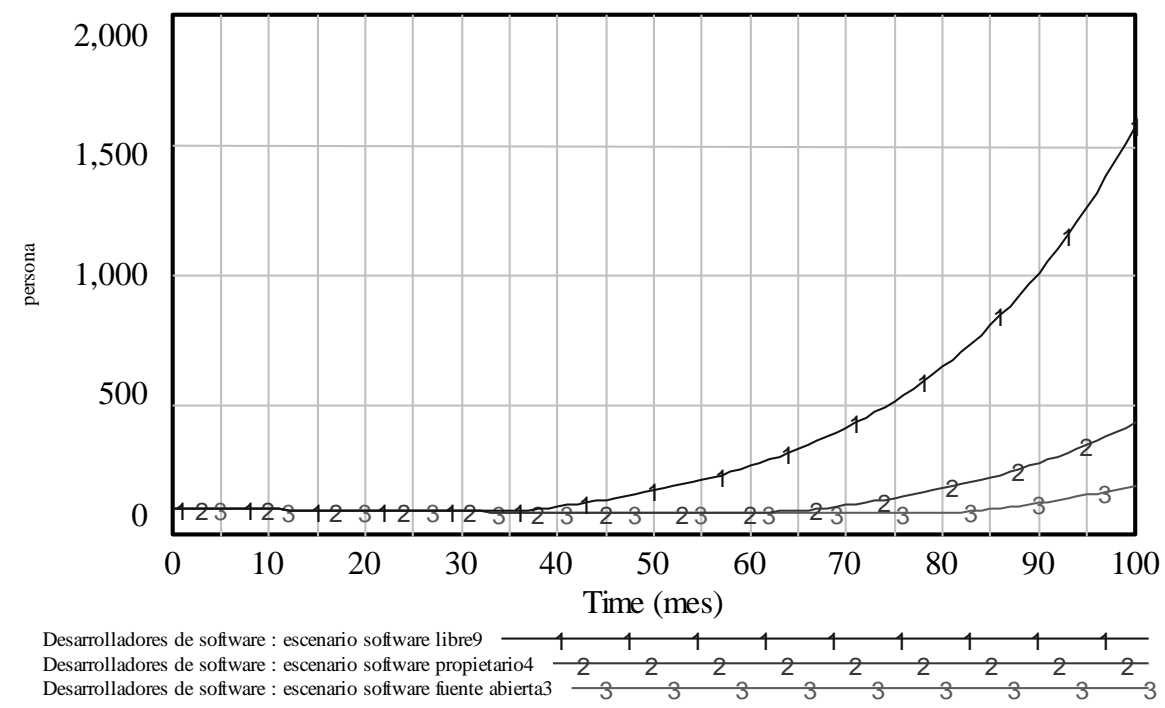

Fig. 7. Desarrolladores de software 


\subsubsection{Aplicaciones en Desarrollo}

La figura 8, de la variable Aplicaciones en desarrollo, deja ver el aumento en formación de desarrolladores en software libre, comparados con los desarrolladores en software propietario y en software de fuente abierta, ya que a mayor desarrolladores en software Libre, habrá mayor número de aplicaciones de software libre iniciadas y por ende en desarrollo. En los primeros meses de simulación no se observan diferencias entre los tres escenarios en cuanto a aplicaciones en desarrollo, a tal punto que hasta el mes 45 ambos escenarios llegan con 4000 aplicaciones aproximadamente en desarrollo. A partir de los meses siguientes, se observan diferencias en los escenarios, diferencia que se mantiene hasta el final de la simulación, es decir hasta el mes 100 . Mostrando en los escenarios que mientras la línea de software libre alcanzó las 38.000 aplicaciones en desarrollo aproximadamente, la línea de software propietario solo alcanzó a llegar a las 10.000 aplicaciones aproximadamente y la de software de fuente abierta solo 5.000 aproximadamente. Lo anterior permite observar un hecho ventajoso y es que teniendo en cuenta la Figura. 6, formación de desarrolladores y la figura 7 desarrolladores de software, que apenas empiezan aparecer estos desarrolladores por allá en el mes 30 y en el 38 respectivamente, en la figura. 8, aplicaciones en desarrollo es diferente, es decir, ya se ven aplicaciones en la etapa de desarrollo desde el primer mes de simulación, lo que indica que los desarrolladores al empezar la etapa de su formación como desarrolladores, empiezan desarrollando aplicaciones de software, lo que hace que aumente el número de aplicaciones iniciadas en ambos escenarios. En resumen, según la figura 8, resultados de la simulación en la variable Aplicaciones en Desarrollo, se observa la coherencia que se ha venido presentando durante la simulación con el escenario de software Libre presentando los mejores resultados.

\subsubsection{Aplicaciones software desarrolladas}

En la figura. 9, se observa la cantidad de aplicaciones desarrolladas de ambos modelos y la diferencia que hay entre un escenario y el otro durante la simulación en el tiempo previsto que fue de 100 meses. Se ve que las primeras aplicaciones de ambos escenarios son desarrolladas a partir del mes 7 aproximadamente, no hay diferencia, solo a partir de del mes 50 se empieza a notar la diferencia en las líneas de crecimiento de los escenarios de software. El Software Libre le tomó una ventaja considerable al Software Propietario y al software de fuente abierta en el incremento de aplicaciones desarrolladas, a tal punto que al final de la simulación, es decir, en el mes 100, la línea de software Libre alcanza las 28.000 aplicaciones desarrolladas aproximadamente, mientras que la línea de software propietario solo llega a 11.000 y la de software de fuente abierta llega a 9.500 aplicaciones desarrolladas aproximadamente. Lo anterior es producto de lo que se ha venido presentando en cada uno de los puntos de análisis en los escenarios del modelo, donde la línea de software Libre le ha tomado ventaja a la del software propietario y a la del software de fuente abierta. Se han desarrollado aplicaciones que según el modelo propuesto son fundamentales en el rendimiento y productividad de las personas, incluso para los mismos desarrolladores y para las empresas productivas de la región. También se observa que ya hay aplicaciones desarrolladas en los primeros siete (7) meses de la simulación, es decir, que una persona antes de culminar su etapa como desarrollador, ya está produciendo aplicaciones de software, principalmente en el escenario de software libre.

En resumen, de lo anterior se observa que Colombia para tener buenas aplicaciones desarrolladas, le debe apostar a la formación de personas como desarrolladores de software en el escenario del modelo que presenta el mayor rendimiento en materia de producción de aplicaciones, que es el escenario del software libre, lo que enriquecerá la industria de software en Colombia y así generar la cantidad de aplicaciones necearías que indica el modelo para contribuir con la mejora de la productividad empresarial.

\subsubsection{Empresas productivas de la región}

En la figura 10, se observa el comportamiento de las empresas productivas de la región durante la simulación, en ella se presenta la forma como se incrementa la creación de las empresas, influenciada por las aplicaciones desarrolladas en ambos modelos, las cuales dependiendo de su pertinencia inciden en la productividad y en el rendimiento operacional de estas empresas, ocasionando que se mantengan activamente en el mercado o el cierre definitivo de sus operaciones por baja productividad. En la figura se presentan los tres escenarios de software, Libre, propietario y fuente abierta, en los cuales se ve una diferencia muy marcada en cuanto a la creación de empresas influenciada por las aplicaciones desarrolladas de acuerdo a su grado de pertinencia. Al final de la simulación, en el escenario de software libre se observa un mayor número de empresas productivas $y$ activas que en el escenario de software Propietario y que en el escenario de software de fuente abierta, es decir, mientras que en la línea de software libre se ven aproximadamente 5.700 empresas productivas y activas en el mercado de la región, en la línea del software propietario sólo se llegó a 1.600 y en la línea de software de fuente abierta se llegó a 750 empresas productivas y activas en el mercado aproximadamente. Demostrando la tendencia que se ha venido presentando durante la simulación, que en cada uno de los puntos donde se comparan los tres escenarios, siempre el escenario de software libre le ha sacado gran ventaja al propietario y al de fuente abierta, tales ventajas como, mayor desarrolladores formados, mayor número de aplicaciones iniciadas, mayor número de aplicaciones en desarrollo, mayor número de aplicaciones desarrolladas y mayor número de empresas activas y productivas en la región. 

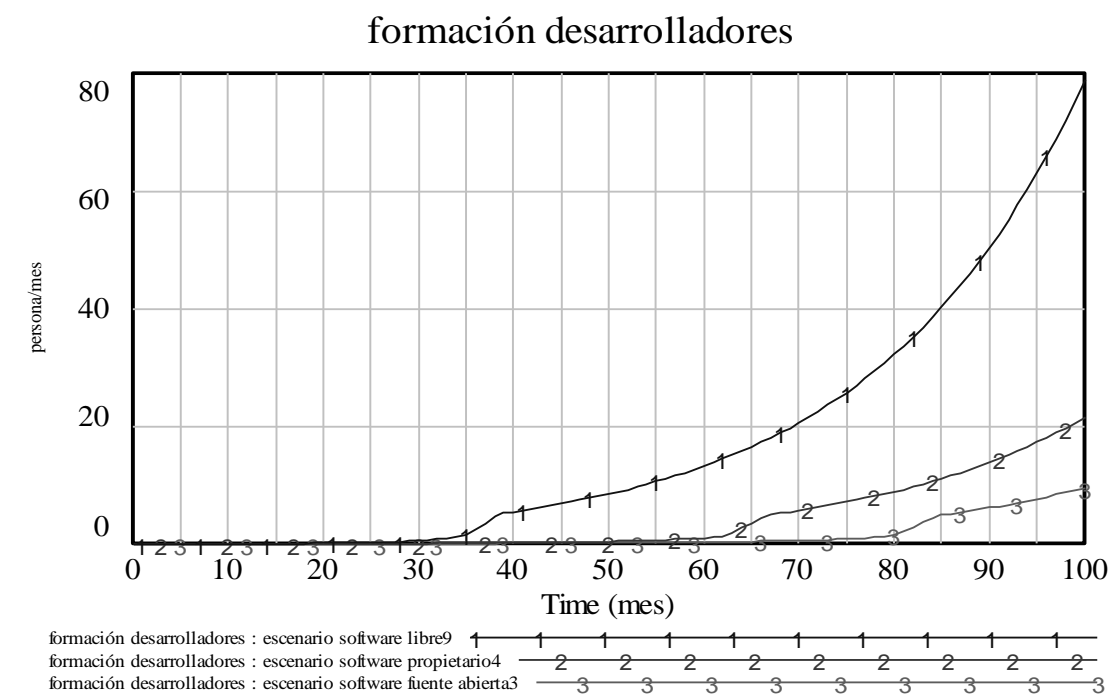

Fig. 8. Aplicaciones en Desarrollo

Aplicaciones software desarrolladas

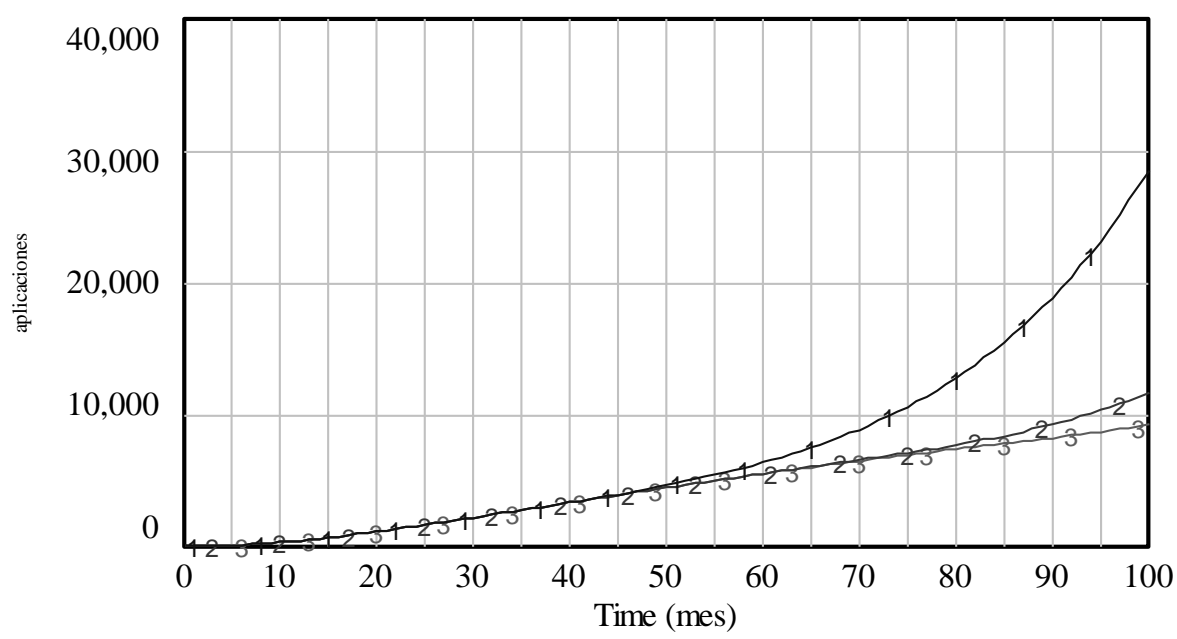

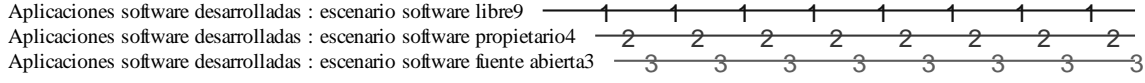

Aplicaciones software desarrolladas : escenario software fuente abierta 13
Fig. 9. Aplicaciones Software Desarrolladas

Empresas productivas de la región

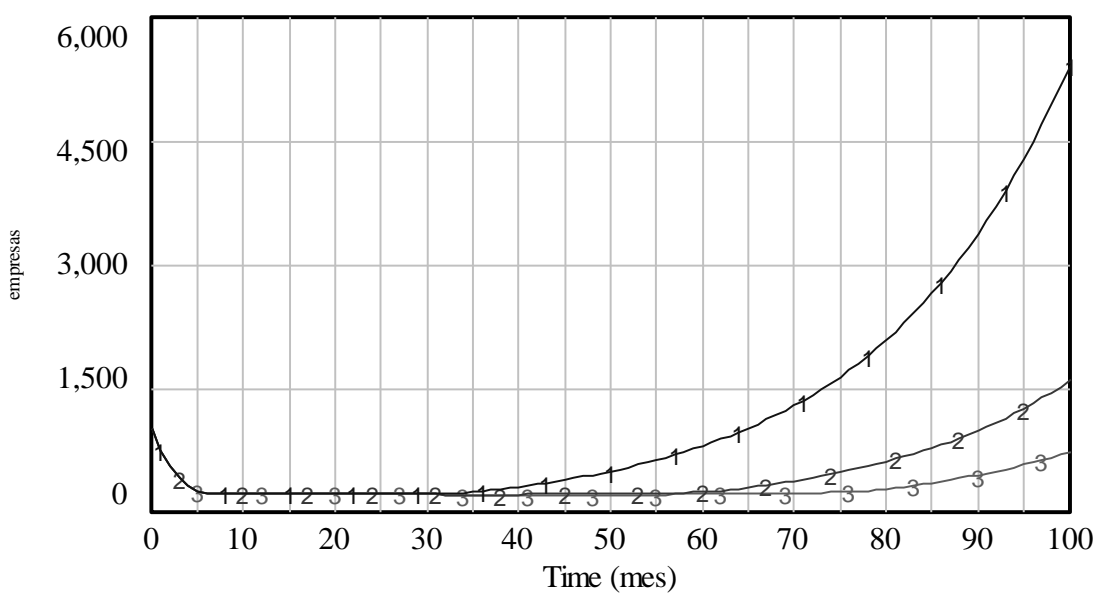

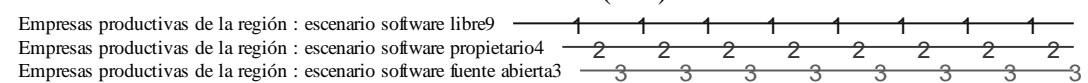

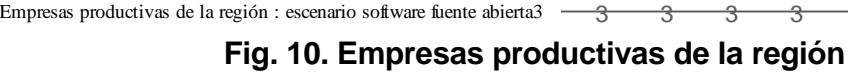




\section{DISCUSIÓN}

Si bien es cierto que hasta la fecha no se han encontrado dentro de la literatura consultada trabajos de este tipo, si hay algunos puntos de partida en algunos trabajos consultados, que se pueden prestar para armar un debate o discusión con los resultados de este trabajo.

Si partimos del hecho que existen cualquier cantidad de investigaciones donde comparan los modelos de software libre, fuente abierta y propietario [12], [15], [18] en donde solo llegan hasta la enumeración de las ventajas y desventajas de ambos modelos y se dan unas conclusiones de acuerdo a los resultados de esas comparaciones, este trabajo ha pretendido ir un poco más allá, donde la idea no es quedarse solo con las conclusiones de los resultados de las comparaciones, sino trascender, tratar de avanzar un poco más a través de los modelos de simulación de la dinámica de sistemas y simular esos resultados de las comparaciones de ambos modelos de software y mostrar a través del tiempo algunas de las implicaciones que se presentan al tratar de separarlos y seleccionar solo uno.

Este trabajo difiere de los trabajos presentados [12], [15], [18], en que mientras que aquí se ha tratado de mostrar las diferencias entre ambos modelo de software haciendo uso de la dinámica de sistemas a través de sus herramientas de modelado con Vensim, lo cual se evalúan unas variables en las que se puede mostrar en cual escenario se maximizan más. En los otros trabajos revisados solo se quedan en las comparaciones y conclusiones de los resultados de esas comparaciones.

En la tabla 1, se presenta una discusión con otros trabajos.

\section{APORTES NOVEDOSOS}

Este trabajo hace un aporte novedoso a las áreas de investigación como son la dinámica de sistemas, al área de la industria del software y a la ciencia como tal, que es la obtención de nuevo conocimiento a través de la generación de un modelo de simulación de la dinámica de sistemas con tres escenarios de software (libre, fuente abierta y propietario), se evalúan unas variables en cada uno de estos escenarios a través de simulaciones, se comparan los resultados de estas simulaciones y se observa en cuál de estos escenarios se producen mejores resultados, los cuales permiten seleccionar uno de los escenarios de software del modelo. Planteándose como una ayuda al aprendizaje desde las diferentes áreas del conocimiento

\section{CONCLUSIONES}

Se obtienen las siguientes conclusiones de este trabajo:

Generación de un modelo de simulación de la dinámica de sistemas con tres escenarios de software (libre, fuente abierta y propietario), se evalúan unas variables en cada uno de estos escenarios mediante simulaciones, se comparan los resultados de estas simulaciones y se observa en cuál de estos escenarios se producen mejores resultados, los cuales permiten seleccionar uno de los escenarios de software del modelo. Planteándose como una ayuda al aprendizaje desde las diferentes áreas del conocimiento

También establece que en el escenario de software libre del modelo planteado, se presentan los mejores resultados de las simulaciones, tales como: Mayor desarrolladores formados, mayor número de aplicaciones iniciadas, mayor número de aplicaciones en desarrollo, mayor número de aplicaciones desarrolladas y mayor número de empresas activas y productivas en la región.

Por lo anterior y teniendo en cuenta los resultados de las simulaciones del modelo en las figuras $6,7,8,9$ y 10 , donde se observa que los mejores resultados de las variables simuladas o evaluadas fueron obtenidos en el escenario del Software Libre, se puede decir que Colombia le puede apostar al escenario del Software Libre.

Este modelo no está diseñado como una herramienta de estimación sino como una herramienta de ayuda al aprendizaje.

\section{TRABAJO FUTURO}

Se propone como trabajo futuro el estudio en campo de la aplicabilidad del modelo con los tres escenarios de software libre, software propietario y software de código abierto, incluyendo otros elementos como: costos, productividad, curvas de aprendizaje, tamaño de la comunidad que usa el software, entre otros. 
Tabla 1. Discusión trabajos de investigación

DISCUSIÓN

Con relación a otros trabajos de investigación que también han abordado los tipos de software libre, propietario o fuente abierta.

\begin{tabular}{|c|c|c|}
\hline TITULO & AUTORES & APORTES \\
\hline $\begin{array}{l}\text { Software libre } \\
\text { software } \\
\text { propietario } \\
\text { Ventajas } \\
\text { desventajas }\end{array}$ & $\begin{array}{l}\text { - Culebro Juárez } \\
\text { Montserrat. } \\
\text { - Gómez Herrera Wendy } \\
\text { Guadalupe. } \\
\text { - Torres Sánchez } \\
\text { Susana. } \\
\text { (Tesis de Maestría) }\end{array}$ & $\begin{array}{l}\text { Establecen comparaciones entre estos modelos, donde se muestra un listado de } \\
\text { las ventajas, desventajas y se dan unas conclusiones. } \\
\text { Se plasman ideas sencillas del concepto del software } \\
\text { Se da una lista de beneficios que podrían producirse en el sector social y se } \\
\text { analiza el marco jurídico del software }\end{array}$ \\
\hline \begin{tabular}{lr} 
Estudio & del \\
comportamiento de \\
la industria del \\
software & en \\
Colombia & ante \\
escenarios & de \\
capacidades & de \\
innovación y & \\
ventajas & \\
comparativas por \\
medio de Dinámica \\
\multicolumn{2}{l}{ de Sistemas } \\
\end{tabular} & $\begin{array}{l}\text { - Karla Cristina Palomino } \\
\text { Zuluaga } \\
\text { (Tesis de Maestría) }\end{array}$ & $\begin{array}{l}\text { características y limitaciones de la industria de software en Colombia } \\
\text { una revisión del estado del arte en el estudio de las capacidades de innovación y } \\
\text { ventajas comparativas desde la perspectiva de la dinámica de sistemas } \\
\text { El modelo propuesto consiste en una adaptación al caso colombiano del modelo } \\
\text { general para el crecimiento y surgimiento de las empresas de software en } \\
\text { países de ingreso tardío }\end{array}$ \\
\hline $\begin{array}{lr}\text { Software } & \\
\text { propietario } & \text { vs } \\
\text { software libre: una } & \text { evaluación re } \\
\text { sistemas integrales } \\
\text { para ra } \\
\begin{array}{lr}\text { automatización de } \\
\text { bibliotecas }\end{array} \\
\end{array}$ & $\begin{array}{l}\text { - Oscar Arriola Navarrete } \\
\text { - Graciela Tecuatl } \\
\text { Quechol } \\
\text { - Guadalupe González } \\
\text { Herrera } \\
\text { (Articulo) }\end{array}$ & $\begin{array}{l}\text { Se evalúan dos Sistemas Integrales de Automatización de Bibliotecas (SIAB) } \\
\text { con el fin de identificar ventajas y desventajas de los sistemas propietarios y los } \\
\text { sistemas libres, con el objetivo de proporcionar información al bibliotecario para } \\
\text { que conozca mayores alternativas que le permitan elegir }\end{array}$ \\
\hline $\begin{array}{l}\text { ¿Colombia debe } \\
\text { apostarle al } \\
\text { software Libre, al } \\
\text { de Fuente Abierta } \\
\text { o al Propietario? }\end{array}$ & $\begin{array}{l}\text { - Luis Alberto Girón } \\
\text { Murillo } \\
\text { (Artículo) }\end{array}$ & $\begin{array}{l}\text { Se genera una revisión documental del tema } \\
\text { Se diseña y se presenta un modelo de simulación de la dinámica de sistemas } \\
\text { con tres escenarios de software (Libre, Propietario y Fuente Abierta). } \\
\text { Se evalúan y se comparan unas variables (desarrolladores de software, } \\
\text { aplicaciones de software, empresas productivas) a través de simulaciones en el } \\
\text { modelo mediante los tres escenarios de software, buscando su optimización } \\
\text { Se realiza la simulación para encontrar la maximización de las variables en cada } \\
\text { uno de los escenarios del modelo } \\
\text { Se describen los resultados obtenidos de las simulaciones y se concluye que: } \\
\text { Al igual que los anteriores trabajos aquí mencionados, este también plantea } \\
\text { comparaciones y conclusiones, pero se diferencia, en que las comparaciones se } \\
\text { establecen desde la productividad de las personas (desarrolladores), } \\
\text { productividad de las empresas y la pertinencia de las aplicaciones de software } \\
\text { desarrolladas. (Ver figuras } 7,9 \text { y 10). } \\
\text { También establece que en el escenario de software libre se presentan los } \\
\text { mejores resultados de las simulaciones, tales como: Mayor desarrolladores } \\
\text { formados, mayor número de aplicaciones iniciadas, mayor número de } \\
\text { aplicaciones en desarrollo, mayor número de aplicaciones desarrolladas y mayor } \\
\text { número de empresas activas y productivas en la región. }\end{array}$ \\
\hline
\end{tabular}




\section{REFERENCIAS}

[1] P. Bastos, F. Silveira y K. Heshusius, "Desafíos y oportunidades de la Industria del Software en América Latina, capítulo Colombia: Desafíos de una industria en formación", Primera edición: mar 2009, pp. $139-170$

[2] S. Monge. "¿Es aplicable el modelo de producción del software libre a contenidos educativos?". RED. Revista de Educación a Distancia. Online [febrero, 2005].

[3] República de Colombia Ministerio de Comunicaciones. Plan nacional de tecnologías de la información y las comunicaciones. Technical report, Ministerio de Comunicaciones, Republica de Colombia, 2008.

[4] Ministerio de Tecnología de la información y las comunicaciones. "Compartel", Online [octubre, 2013].

[5] Red Hat Corporation. "Open source activity map", Online [Mayo, 2010].

[6] L. F. Medina, R. A. Pava. "Propuesta de un marco de trabajo para la investigación formal en Software libre y/o de código abierto", Corporación Universitaria Minuto de Dios, 2010.

[7] J. M. Calvo. "El Software Libre en Colombia, ¿El Software Libre está listo para ser utilizado en la empresa? ¿Las empresas en Colombia están utilizando Software Libre? ¿Para qué lo están utilizando?", Revista ACIS. Online [noviembre, 2004].

[8] E. S. Raymond. "La catedral y el bazar". 1998. Online [octubre, 2012].

[9] R. M. Stallman. "Software libre para una sociedad libre". Madrid: Edición Traficantes de Sueños versión 10, 2004.
[10] R. M. Stallman. "General Public License. GNU. Free Software Foundation, Inc. February 1989, version 1.0". Online [septiembre, 2013]

[11] J. González, J. S. Pascual, G. Robles. "Introducción al software libre". UOC. Madrid: Primera edición. Online [febrero, 2008].

[12] M. Culebro, W. G. Gómez, S. Torres. "Software libre vs software propietario: ventajas y desventajas", México: Creative Commons, 2006. Online [junio, 2010].

[13] A. M. Delgado, R. O. Cuello. "La promoción del uso del software libre por parte de las universidades". RED. Revista de Educación a Distancia, número 17. Online [agosto, 2013].

[14] M. Carranza. "Problemática jurídica del software libre", Buenos Aires: LexisNexis, 2004, p. 42.

[15] K. C. Palomino. "Estudio del Comportamiento de la Industria del Software en Colombia ante Escenarios de Capacidades de Innovación y Ventajas Comparativas Por Medio de Dinámica de Sistemas", UNAL Facultad de Minas, 2011. Online [abril, 2013].

[16] J. Torrealdea. "Ciencias de la Computación e Inteligencia Artificial". Online [diciembre, 2013].

[17] I. M. Santa Catalina. Tesis Doctoral: "Modelo de Dinámica de Sistemas para la Implantación de Tecnologías de la Información en la Gestión Estratégica Universitaria". Universidad del País Vasco: Euskal Herriko Unibertsitatea. Donostia - San Sebastián, 2010.

[18] O. Arriola, G. Tecuatl y G. González. "Software Propietario vs Software libre: Una evaluación de sistemas integrales para la automatización de bibliotecas" Online [octubre, 2013]. 